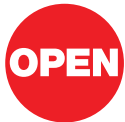

SUBJECT AREAS: BREEDING

EVOLUTION

ECOLOGY

EPIGENETICS

Received

21 February 2012

Accepted

4 April 2012

Published

26 April 2012

Correspondence and requests for materials should be addressed to J.A.Z. (jaz@unr.edu)

\section{From father to son: transgenerational effect of tetracycline on sperm viability}

\author{
Jeanne A. Zeh, Melvin M. Bonilla, Angelica J. Adrian, Sophia Mesfin \& David W. Zeh
}

Department of Biology and Program in Ecology, Evolution and Conservation Biology, University of Nevada, Reno, NV 89557, USA.

The broad-spectrum antibiotic tetracycline is used in animal production, antimicrobial therapy, and for curing arthropods infected with bacterial endosymbionts such as Wolbachia. Tetracycline inhibits mitochondrial translation, and recent evidence indicates that male reproductive traits may be particularly sensitive to this antibiotic. Here, we report the first multi-generation investigation of tetracycline's effects on ejaculate traits. In a study of the pseudoscorpion, Cordylochernes scorpioides, in which siblings were randomly assigned to control and tetracycline treatments across replicate full-sibling families, tetracycline did not affect body size in either sex, female reproduction or sperm number. However, tetracycline-treated males exhibited significantly reduced sperm viability compared to control males, and transmitted this toxic effect of tetracycline on sperm to their untreated sons but not to their F2 grandsons. These results are consistent with tetracycline-induced epigenetic changes in the male germline, and suggest the need for further investigation of transgenerational effects of tetracycline on male reproductive function.

espite more than six decades of therapeutic and agricultural use that has resulted in the evolution of widespread bacterial resistance ${ }^{1}$, the broad-spectrum antibiotic tetracycline is still commonly used as an additive in animal feed ${ }^{2}$ and as an accessible antimicrobial therapy in developing countries ${ }^{3}$. Tetracycline is easily isolated from Streptomyces fermentation, and its extensive use stems from both its low cost and its effectiveness against a diverse range of microorganisms ${ }^{4}$. Unlike penicillin and other beta-lactam antibiotics that target the bacterial cell wall, tetracycline is effective against intracellular pathogens (e.g., Chlamydiae, Rickettsiae and mycoplasmas), in which the cell wall is deficient or absent ${ }^{5}$. There is growing evidence that rickettsial bacteria, most notably Wolbachia, are pervasive inhabitants of insect cytoplasm ${ }^{6,7}$, and assessing Wolbachia effects on host phenotype typically involves treatment with tetracycline in order to cure cellular endosymbiont infection. An implicit but infrequently tested assumption is that antibiotic treatment has negligible impacts on the morphological, life history and/or reproductive traits under investigation ${ }^{8}$.

Tetracycline is a protein synthesis inhibitor that acts by blocking attachment of amino acid-bearing tRNAs to the $30 \mathrm{~S}$ subunit of prokaryote ribosomes ${ }^{9}$. As descendents of ancient $\alpha$-proteobacteria ${ }^{10}$, mitochondria possess bacteria-type ribosomes, and tetracycline is known to inhibit mitochondrial protein synthesis ${ }^{11}$. With mitochondria playing a critical role in spermatogenesis and sperm motility ${ }^{12}$, male reproductive function appears to be particularly sensitive to antibiotics. For example, in Tilapia, exposure of sperm to high concentrations of antibiotics decreased both sperm motility and mitochondrial function ${ }^{13}$. In rats, tetracycline has been shown to produce morphological abnormalities in sperm, as well as reduced sperm counts, motility and viability ${ }^{3}$. In vitro studies of human sperm have demonstrated similar negative effects of tetracycline on motility and viability ${ }^{14}$. Still to be determined, however, is whether the detrimental effects of antibiotics on reproductive traits can be perpetuated across generations. It is becoming increasingly apparent that environmental factors, including diet and endocrine disrupting chemicals, can induce epigenetic changes that are transmitted to offspring through the male germ line ${ }^{15}$.

Here, in a study of the pseudoscorpion, Cordylochernes scorpioides, we report the first multigenerational investigation of the effects of tetracycline on sperm count and sperm viability. The biology of this pseudoscorpion is ideally suited for investigating the fitness consequences of tetracycline treatment. Males transfer sperm to females in discrete packets via a stalked spermatophore deposited on the substrate ${ }^{16}$. External spermatophore deposition provides a window on ejaculate characteristics that is lacking in species that transfer sperm directly. Matings can be interrupted immediately following spermatophore deposition, and the sperm packet (Fig. 1a) collected for assessment of sperm quantity and quality ${ }^{17}$. Females are viviparous, and nourish developing embryos in an external, transparent brood sac (Fig. 1b), overlying the genital aperture ${ }^{18,19}$. This "external womb" form of viviparity makes possible non-invasive monitoring of female reproductive status and embryological development ${ }^{20,21}$. 
a

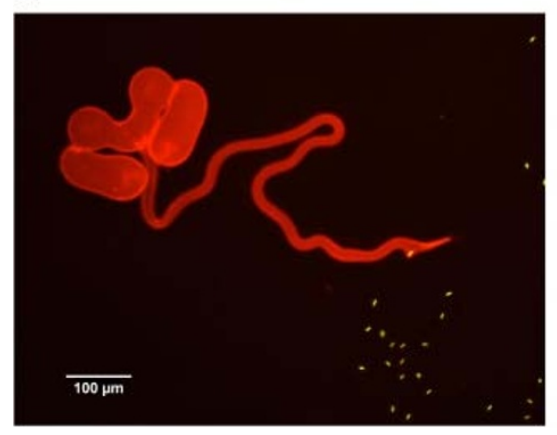

b

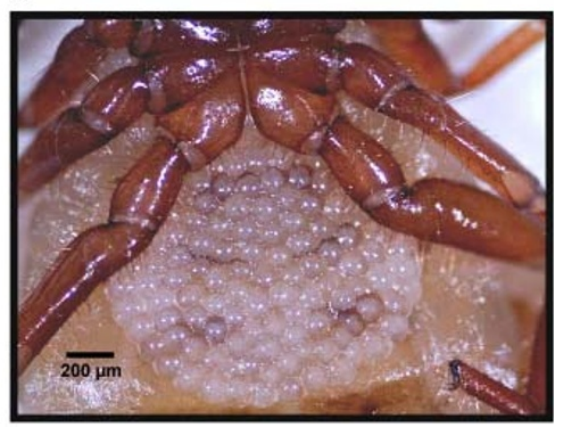

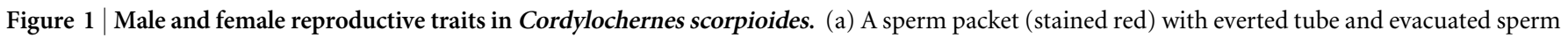
(stained green). (b) Ventral view of a female carrying a brood sac of approximately 100 early-stage embryos.

To assess potential direct and indirect (transgenerational) effects of tetracycline on male reproductive function, sperm number and sperm viability were assayed across three generations. In the F0 generation directly exposed to tetracycline, we used a split-brood design, in which offspring from replicate full-sibling families were randomly assigned at birth to control $(\mathrm{C})$ and tetracycline $(\mathrm{T})$ treatments for rearing through the adult stage. Males and females from the F0 generation were subsequently paired in all four possible mat-

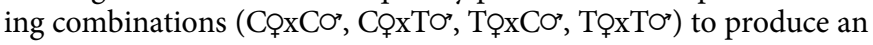
untreated F1 generation. Adult F1 TT and CC males were then outcrossed to untreated females from a large laboratory population in order to determine whether paternal treatment effects on sperm traits were perpetuated in F2 offspring.

Whereas tetracycline had no discernable effects on female reproductive function or body size in either sex, our results indicate that tetracycline-treated males exhibited significantly reduced sperm viability, and transmitted this toxic effect of tetracycline on sperm to their untreated sons but not to their grandsons. Such cryptic, multigenerational effects of antibiotics on sperm have potentially important implications for studies of arthropod model organisms infected with cellular endosymbionts, and warrant investigation as a possible factor contributing to the decades-long decline in human ejaculate quality.

\section{Results}

Direct tetracycline treatment of F0 males exerted a significantly detrimental effect on the viability of their sperm compared to that of control males $\left(F_{1,30}=9.34, P=0.0047\right.$; Fig. $\left.2 \mathrm{a}\right)$. However, no other traits in F0 males or females were significantly affected by tetracycline treatment (sperm number, $F_{1,30}=0.02, P=0.8935$; male cephalothorax length, $F_{1,30}=0.00 ; P=0.9962$ female cephalothorax length, $F_{1,30}=0.21, P=0.6477$; Fig. 3a).

Analysis of ejaculate characteristics in the $\mathrm{F} 1$ generation revealed a significant negative effect of tetracycline treatment of F0 sires on the viability of their sons' sperm (sire effect, $F_{1,22}=6.49, P=0.0184$ ) but no effect of dam treatment $\left(F_{1,22}=2.26 ; P=0.1469\right)$ and a nonsignificant dam $\mathrm{x}$ sire interaction $\left(F_{1,22}=2.48, P=0.1294\right)$. However, the lowest sperm viability did occur in male offspring of the tetracycline dam $\mathrm{x}$ tetracycline sire crosses (Fig. 2b). Cross type had no significant effect on sperm number (sire effect, $F_{1,29}=0.06$, $P=0.8049$; dam effect, $F_{1,29}=0.11, P=0.7430$; sire $\mathrm{x}$ dam interaction, $\left.F_{1,29}=0.09, P=0.7655\right)$.

Non-significant main and interaction effects of sire and dam treatments were also observed for early-stage embryo counts (sire effect, $F_{1,37}=0.02, P=0.8965$; dam effect, $F_{1,37}=2.25, P=0.1421$; sire $\mathrm{x}$ dam interaction, $F_{1,37}=3.35, P=0.0753$; Fig. $3 \mathrm{~b}$ ) and number of protonymphs born (sire effect, $F_{1,38}=0.02, P=0.9032$; dam effect, $F_{1,38}=0.66, P=0.4204$; sire $\mathrm{x}$ dam interaction, $F_{1,38}=1.66, P=$ 0.2056; Fig. 3c). Females that failed to become gravid were excluded from these analyses. However, including zero embryo and proto- nymph scores for non-gravid females did not qualitatively change the results (data not shown). In addition, there were no significant main or interaction effects of sire and dam treatments on male body size (sire effect, $F_{1,29}=0.53, P=0.4735$; dam effect, $F_{1,29}=0.37, P=$ 0.5489; sire x dam interaction, $F_{1,29}=3.76, P=0.0621$ ) or female body size (sire effect, $F_{1,29}=1.41, P=0.2443$; dam effect, $F_{1,29}=$ $0.00, P=0.9896$; sire $\mathrm{x}$ dam interaction, $\left.F_{1,29}=0.08, P=0.7754\right)$.

The significant reduction of sperm viability in F0 tetracyclinetreated males and their untreated F1 sons was not transmitted to the F2 generation (Fig. 2c). The offspring of TT males did not differ significantly from the sons of CC males in either sperm viability $\left(F_{1,23}\right.$ $=0.01, P=0.9057)$ or sperm number $\left(F_{1,23}=0.03, P=0.8710\right)$.

\section{Discussion}

In this study, tetracycline had no detrimental consequences for sperm number, female reproduction or body size in either sex. By contrast, tetracycline-treated males exhibited significantly reduced sperm viability, and transmitted this toxic effect of tetracycline on sperm to their untreated sons but not to their F2 grandsons. The transient nature of this paternally-inherited effect is consistent with tetracycline-induced epigenetic changes in the male germline. Exposure to epigenome-modifying agents during critical phases of development can result in transmission of altered epigenetic states, involving changes in DNA methylation, histone modifications or non-coding RNAs, across one to several generations ${ }^{15}$. Wallace \& $\mathrm{Fan}^{22}$ have proposed that mitochondria play a critical role in mediating such epigenetic effects, particularly with respect to DNA methylation, and a recent study of mice exposed to particulate air pollutants has provided direct experimental evidence for this hypothesis $^{23}$. Interestingly, in contrast to most arthropods ${ }^{24}$, preliminary research in our laboratory indicates that a significant fraction of the C. scorpioides genome is $\mathrm{CpG}$ methylated. We hypothesize that adverse effects of tetracycline on mitochondria in testicular tissue of F0 tetracycline-treated males caused oxidative damage that affected DNA methylation via the S-adenosyl-methionine pathway, resulting in heritable epigenetic modifications to the male germline. Epigenetically-based phenotypic disruption seems particularly plausible, since normal sperm viability was restored in F2 C. scorpioides males.

It is important to note that alternative mechanisms could explain our findings. For example, the negative effects on sperm viability could be the result of tetracycline-induced changes in the C. scorpioides microbiome. However, this explanation seems unlikely. As strict carnivores that feed by injecting digestive enzymes into their prey $^{18}$, pseudoscorpions do not depend on symbiotic bacteria or unicellular eukaryotes for cellulose digestion. In addition, microbial populations were likely to have been reestablished in the untreated F1 generation, and microbiome-mediated effects cannot easily account for significant paternal but not maternal effects on sperm viability in F1 males. 
a

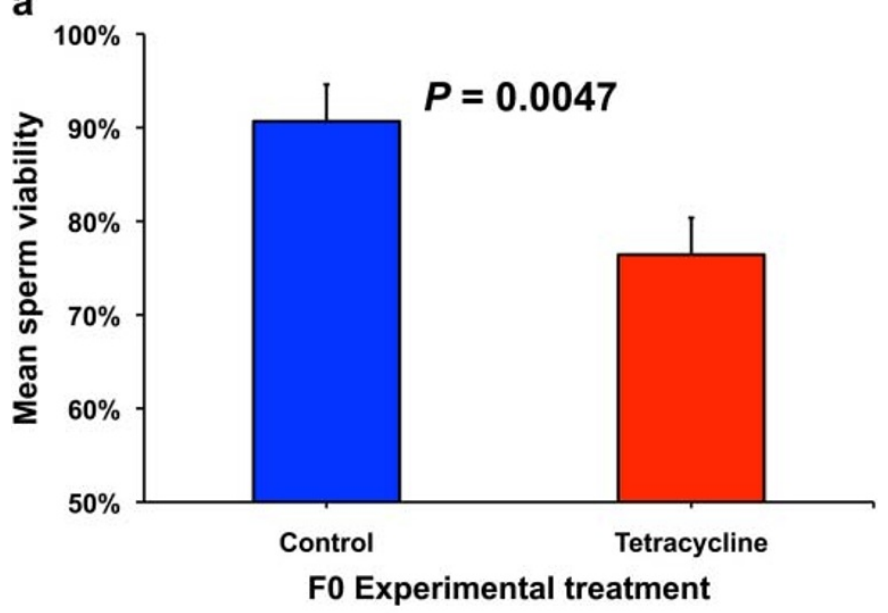

b
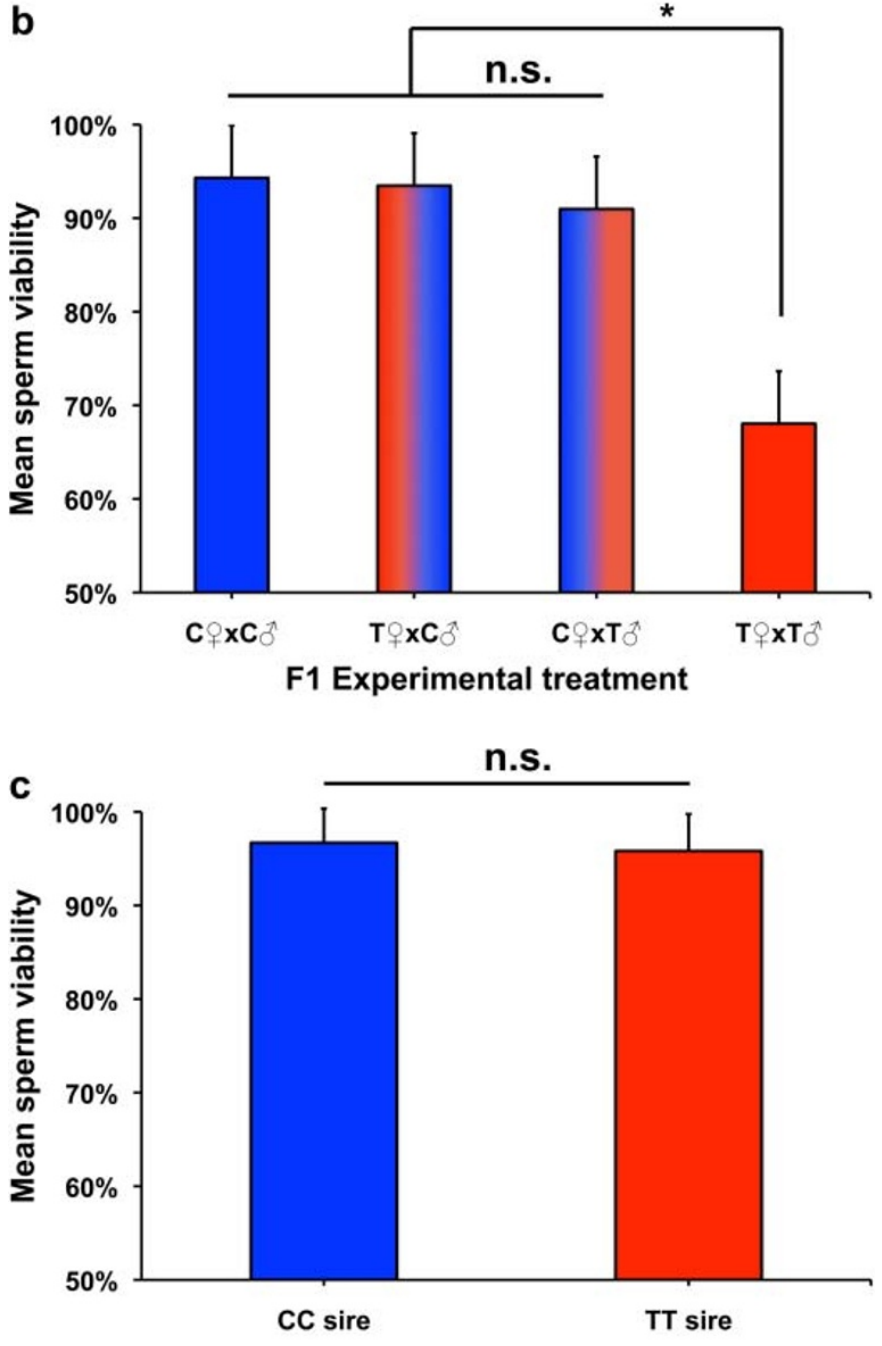

F2 Experimental treatment

Figure $2 \mid$ Tetracycline effects on sperm viability across three generations. (a) Mean sperm viability ( + standard error) in control (C) and tetracycline-treated (T) males in the F0 (directly treated) generation. (b) Mean sperm viability in F1 untreated male offspring from the four cross types. (c) Mean sperm viability in the F2 sons of CC and TT males outcrossed to females from the laboratory population. Two-tailed $P$ values were determined using a General Linear Mixed Model fit to a binomial response variable (proportion live sperm). In (b), multiple comparison $P$ values were calculated using Tukey's HSD (honestly significant difference) test. a

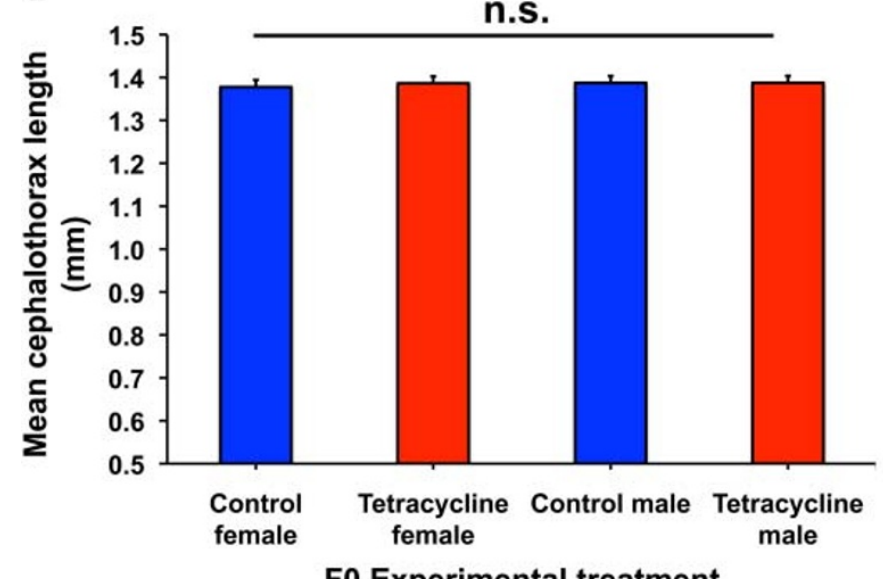

b

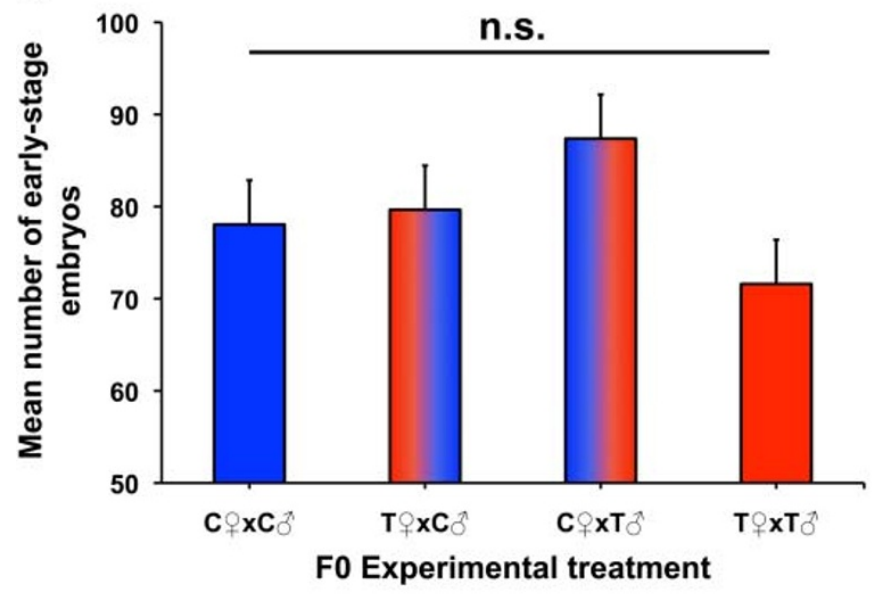

C

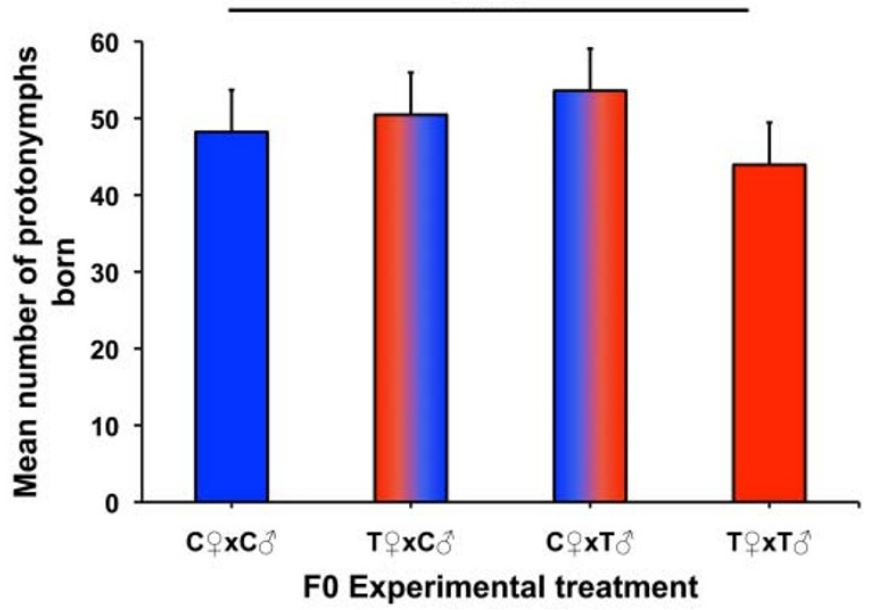

Figure $3 \mid$ Absence of tetracycline effects on body size and female reproduction in the F0 generation. Means (+ standard error) for (a) cephalothorax length. (b) number of early-stage embryos. (c) number of protonymphs born.

The transgenerational effect of antibiotics on sperm reported here has potentially important implications for studies of arthropod species infected with cellular endosymbionts such as Wolbachia. A recent estimate suggests that these obligate intracellular bacteria are present in two-thirds of insect species ${ }^{7}$, and they also occur in nematodes, amphipods, isopods and arachnids ${ }^{25}$. As with other genetic elements present in the cytoplasm, Wolbachia are transmitted to 
offspring only through eggs, and can therefore enhance their fitness by biasing host sex ratio in favor of females ${ }^{26}$. Wolbachia employ a variety of mechanisms for manipulating host sex ratio, including feminization, parthenogenesis induction in haplo-diploid species, and killing male embryos early in development ${ }^{25}$. Assessing Wolbachia effects on host phenotype usually involves comparing infected individuals with individuals treated with tetracycline to cure the cellular endosymbiont infection (e.g., ${ }^{19}$ ). Implicit in this approach is the assumption that tetracycline has little or no effect on the survival, morphology or reproduction of treated individuals and their descendents. While this assumption proved valid for C. scorpioides females, it was strongly violated for male reproductive function across two generations in our study. Taken in conjunction with the recent finding that tetracycline reduced mitochondrial OXPHOS activity in Drosophila simulans two generations after treatment ${ }^{8}$, our results suggest that transgenerational effects of tetracycline may be more common in arthropods than is currently appreciated.

The increasing evidence for pernicious effects of antibiotics on spermatogenesis can provide a parsimonious explanation for the marked decline in human ejaculate quality over the last 60 years ${ }^{27}$. Although declining sperm density has generally been attributed to increased exposure to pesticides and other endocrine disrupting chemicals ${ }^{28}$, the onset of this decline ${ }^{29}$ also coincided with the introduction and widespread use of antibiotics in the 1950s. In one of the few studies that have statistically evaluated multiple factors potentially contributing to male subfertility ${ }^{30}$, recent use of antibiotics, primarily penicillin and doxycycline (a derivative of the tretracycline group), outranked pesticide exposure, welding and smoking as the single most significant risk factor for oligospermia (low sperm count). Taken together, the results of animal and human studies indicate that antibiotic use should be given serious consideration as a factor contributing to human male subfertility, and that further investigation of the direct and transgenerational effects of antibiotics on male reproductive function in model organisms is clearly warranted.

\section{Methods}

Pseudoscorpions and experimental methods. Pseudoscorpions for this study were drawn from a large laboratory population established from 350 C. scorpioides individuals collected in 2006 and 2008 from six locations spanning a 60-km region in central Panamá ${ }^{21}$. In the F0 generation that received direct exposure to tetracycline, we used a split-brood design involving 21 full-sibling families, in which 24 protonymphs (first-stage nymphs) from each family were randomly assigned at birth, 12 to a tetracycline treatment $(\mathrm{T})$ and 12 to an untreated, control treatment $(\mathrm{C})$. Protonymphs were reared to adults in individual vials, and provisioned weekly with Drosophila melanogaster larvae. After molting to the adult stage, offspring were fed Tribolium confusum larvae. Tetracycline was administered to pseudoscorpions by rearing their prey on medium supplemented with tetracycline hydrochloride (Sigma). For D. melanogaster, 4-24® instant Drosophila medium (Carolina Biological) was supplemented with tetracycline at a concentration of $0.52 \mathrm{mg} / \mathrm{ml}$. For T. confusum, larvae were reared in an $8: 1(\mathrm{v} / \mathrm{v})$ mixture of wheat flour and brewer's yeast containing a $0.2 \%$ concentration $(\mathrm{w} / \mathrm{w})$ of tetracycline hydrochloride.

Cephalothorax length, a representative measure of body $\operatorname{size}^{20}$, was determined, as described elsewhere ${ }^{21}$, for four adult individuals from each of the family by treatment by sex categories. Two $\mathrm{C}$ and two T males from each family were paired with nonexperimental, virgin females and sperm packets collected for assessment of sperm number and viability. Sperm packets were ruptured in $9 \mu \mathrm{L}$ of phosphate buffered saline (140 mM NaCl; $2.7 \mathrm{mM} \mathrm{KCl} ; 8.1 \mathrm{mM} \mathrm{Na} 2 \mathrm{HPO} 4 ; 8.1 \mathrm{mM} \mathrm{KH} 2 \mathrm{PO} 4$; $\mathrm{pH}=$ 7.4) to disperse the sperm. After the addition of $1 \mu \mathrm{L}$ of a 1:50 dilution of $1 \mathrm{mM}$ SYBR 14 , the sperm solution was incubated in the dark for $10 \mathrm{~min}$ before adding $1 \mu \mathrm{L}$ of $2.4 \mathrm{mM}$ propidium iodide (Invitrogen Live/Dead Sperm Viability Kit). The sample was then incubated in the dark for a further $10 \mathrm{~min}$. SYBR 14 is a membrane-permeant, nucleic-acid stain that labels live sperm with green fluorescence, while propidium iodide is membrane-impermeant and labels membrane-compromised (inviable) sperm with red fluorescence. Each $11-\mu \mathrm{L}$ stained sample was transferred to a hemocytometer, and viewed under a range of magnifications (40X to 400X), using an Olympus BX51 fluorescence microscope equipped with an EM510 dual band pass fluorescence filter cube to enable simultaneous counting of SYBR 14 and propidium iodide stained sperm.

To assess tetracycline effects on female reproduction, F0 control and treated virgin females and males were mated to yield 16 replications of each of the four cross types, i.e., control female $\mathrm{x}$ control male (CC), tetracycline female $\mathrm{x}$ control male (TC), control female $\mathrm{x}$ tetracycline male (CT) and tetracycline female $\mathrm{x}$ tetracycline male (TT). Each pair was placed in a $28-\mathrm{mm}$ mating arena, and given a $40 \mathrm{~min}$ mating opportunity. Males typically produce a spermatophore within 7 min of encountering a female, and previous research on C. scorpioides, involving several hundred videotaped matings, indicates that $98 \%$ of virgin females are sexually receptive ${ }^{16,20}$. After mating, C. scorpioides females exhibit one of the three possible outcomes: (i) failure to produce a brood of embryos; (ii) production of a brood of embryos but subsequent spontaneous abortion of the entire brood, or (iii) brood production and carrying of embryos to term, with all nymphs birthing simultaneously. Commencing on the fifth day following mating, each female was monitored until she gave birth to a brood of nymphs, spontaneously aborted her brood or failed to produce a brood within $30 \mathrm{~d}$. Analysis of the lifetime reproductive success of more than 200 C. scorpioides females indicates that females failing to produce a brood within three weeks of mating never become gravid $^{20}$. Females that did produce broods were carefully removed from their vials as soon as individual embryos became clearly distinguishable, and their brood sacs photographed for embryo counting, as described elsewhere ${ }^{19}$. The females were then returned to their vials, and monitored until they either gave birth to a first brood of nymphs or spontaneously aborted the brood sac. During gestation, females remain in a silken nest constructed on the vial wall and brood development could therefore be monitored without further disturbance to the female ${ }^{20}$. Within $48 \mathrm{~h}$ of birth, nymphs were removed from the nest and counted.

To investigate transgenerational effects of tetracycline treatment, offspring from eight replications of each cross type were reared to adults on tetracycline-free prey for assessment of F1 body size ( $n=212$ males; 188 females) and ejaculate characters $(n=$ 16 CC; 16 CT; 15 TC and 16 TT males). Finally, to determine whether the significant effect of sire treatment on sperm viability in the F1 (see Results) was perpetuated in the F2 generation, the offspring of $24 \mathrm{~F} 1 \mathrm{TT}$ and $24 \mathrm{CC}$ males outcrossed to untreated females from the laboratory population were reared on tetracycline-free prey, and sperm number and viability quantified for 32 and 27 sons of TT and CC males, respectively.

Statistical analyses. To analyze treatment effects on morphology, ejaculate traits and female reproduction, we used a general linear mixed model (GLMM), as implemented in SAS ${ }^{31}$. To avoid pseudoreplication, full-sibling family identity was included in the models as a random effect. Cephalothorax length and sperm number were approximately normally distributed, and GLMMs for analyzing these response variables incorporated a Gaussian distribution, an identity link function, a Laplace maximum likelihood approximation, and the SAS containment degrees of freedom $\operatorname{method}^{31}$. Since sperm viability was assessed as the proportion of live sperm, we analyzed this response variable, using the GLIMMIX logit link function to fit a binomial response variable ${ }^{31}$. The $\mathrm{P}$ values for all statistical tests are two-tailed.

1. Zakeri, B. \& Wright, G. D. Chemical biology of tetracycline antibiotics. Biochem. Cell Biol. 86, 124-136 (2008).

2. Silbergeld, E. K., Graham, J. \& Price, L. B. Industrial food animal production, antimicrobial resistance, and human health. Annu. Rev. Publ. Health 29, 151-169 (2008).

3. Farombi, E. O., Ugwuezunmba, M. C., Ezenwadu, T. T., Oyeyemi, M. O. \& Ekor, M. Tetracycline-induced reproductive toxicity in male rats: effects of vitamin C and N-acetylcysteine. Exp. Toxicol. Pathol. 60, 77-85 (2008).

4. Roberts, M. C. Tetracycline therapy: update. Clin. Infect. Dis. 36, 462-467 (2003).

5. Ryan, K., Ray, C. G., Ahmad, N., Drew, W. L. \& Plorde, J. Sherris Medical Microbiology, Fifth Edition. (USA: McGraw-Hill Medical, 2010).

6. Duron, O., Bouchon, D., Boutin, S., Bellamy, L., Zhou, L. Q., Engelstadter, J. \& Hurst, G. D. The diversity of reproductive parasites among arthropods: Wolbachia do not walk alone. BMC Biol. 6, 27 (2008).

7. Hilgenboecker, K., Hammerstein, P., Schlattmann, P., Telschow, A. \& Werren, J. H. How many species are infected with Wolbachia? - a statistical analysis of current data. FEMS Microbiol. Lett. 281, 215-220 (2008).

8. Ballard, J. W. O. \& Melvin, R. G. Tetracycline treatment influences mitochondrial metabolism and mtDNA density two generations after treatment in Drosophila. Insect Mol. Biol. 16, 799-802 (2007).

9. Brodersen, D. E., Clemons, W. M., Carter, A. P., Morgan-Warren, R. J., Wimberly, B. T. \& Ramakrishnan, V. The structural basis for the action of the antibiotics tetracycline, pactamycin, and hygromycin B on the 30 S ribosomal subunit. Cell 103, 1143-1154 (2000).

10. Thrash, J. C., Boyd, A., Huggett, M. J., Grote, J., Carini, P., Yoder, R. J., Robbertse, B., Spatafora, J. W., Rappe, M. S. \& Giovannoni, S. J. Phylogenomic evidence for a common ancestor of mitochondria and the SAR11 clade. Sci. Rep. 1, 13 (2011).

11. Zhang, L., Ging, N. C., Komoda, T., Hanada, T., Suzuki, T. \& Watanabe, K. Antibiotic susceptibility of mammalian mitochondrial translation. FEBS Lett. 579, 6423-6427 (2005).

12. Zeh, J. A. \& Zeh, D. W. Maternal inheritance, sexual conflict and the maladapted male. Trends Genet. 21, 281-286 (2005).

13. Segovia, M., Jenkins, J. A., Paniagua-Chavez, C. \& Tiersch, T. R. Flow cytometric evaluation of antibiotic effects on viability and mitochondrial function of refrigerated spermatozoa of Nile tilapia. Theriogenol. 53, 1489-1499 (2000).

14. Hargreaves, C. A., Rogers, S., Hills, F., Rahman, F., Howell, R. J. S. \& Homa, S. T. Effects of co-trimoxazole, erythromycin, amoxycillin, tetracycline and chloroquine on sperm function in vitro. Hum. Reprod. 13, 1878-1886 (1998).

15. Skinner, M. K., Manikkam, M. \& Guerrero-Bosagna, C. Epigenetic transgenerational actions of environmental factors in disease etiology. Trends Endocrin. Met. 21, 214-222 (2010). 
16. Zeh, J. A., Newcomer, S. D. \& Zeh, D. W. Polyandrous females discriminate against previous mates. Proc. Natl. Acad. Sci. USA 95, 13732-13736 (1998).

17. Bonilla, M. M., Zeh, D. W., White, A. M. \& Zeh, J. A. Discriminating males and unpredictable females: males bias sperm allocation in favor of virgin females. Ethology 117, 740-748 (2011).

18. Weygoldt, P. The Biology of Pseudoscorpions. (Cambridge, MA: Harvard University Press, 1969).

19. Koop, J. L., Zeh, D. W., Bonilla, M. M. \& Zeh, J. A. Reproductive compensation favours male-killing Wolbachia in a live-bearing host. Proc. R. Soc. Lond. B 276, 4021-4028 (2009).

20. Newcomer, S. D., Zeh, J. A. \& Zeh, D. W. Genetic benefits enhance the reproductive success of polyandrous females. Proc. Natl. Acad. Sci. USA 96, 10236-10241 (1999).

21. Zeh, J. A., Bonilla, M. M., Su, E. J., Padua, M. V., Anderson, R. V., Kaur, D., Yang, D.-S. \& Zeh, D. W. Degrees of disruption: projected temperature increase has catastrophic consequences for reproduction in a tropical ectotherm. Global Change Biol. (2012) doi: 10.1111/j.1365-2486.2012.02640.x

22. Wallace, D. C. \& Fan, W. W. Energetics, epigenetics, mitochondrial genetics. Mitochondrion 10, 12-31 (2010).

23. Soberanes, S. et al. Particulate matter air pollution induces hypermethylation of the p16 promoter via a mitochondrial ROS-JNK-DNMT1 pathway. Sci. Rep. 2, 275 (2011).

24. Jeltsch, A. Phylogeny of methylomes. Science 328, 837-838 (2010).

25. Werren, J. H., Baldo, L. \& Clark, M. E. Wolbachia: master manipulators of invertebrate biology. Nat. Rev. Microbiol. 6, 741-751 (2008).

26. Cosmides, L. M. \& Tooby, J. Cytoplasmic inheritance and intragenomic conflict. J. Theor. Biol. 89, 83-129 (1981).

27. Swan, S. H., Elkin, E. P. \& Fenster, L. The question of declining sperm density revisited: an analysis of 101 studies published 1934 -1996. Envir. Health Persp. 108, 961-966 (2000).

28. Sharpe, R. M. Environmental/lifestyle effects on spermatogenesis. Phil. Trans. $R$. Soc. B 365, 1697-1712 (2010).
29. Carlsen, E., Giwercman, A., Keiding, N. \& Skakkebaek, N. Evidence of decreasing quality of semen during past 50 years. Brit. Med. J. 305, 609-613 (1992).

30. Wong, W. Y., Zielhuis, G. A., Thomas, C. M. G., Merkus, H. M. W. M. \& SteegersTheunissen, R. P. M. New evidence of the influence of exogenous and endogenous factors on sperm count in man. Eur. J. Obstet. Gyn. Reprod. Biol. 110, 49-54 (2003).

31. SAS. The SAS System for Windows, Release 9.2, The GLIMMIX Procedure. (Cary, NC: SAS Institute, 2008).

\section{Acknowledgments}

We thank La Autoridad Nacional del Ambiente (A.N.A.M.) for permission to collect pseudoscorpions in Panamá and the Smithsonian Tropical Research Institute for extensive logistical support. This research was supported by grants from the National Geographic Society and the National Science Foundation (DEB-0721226) to J.A.Z. \& D.W.Z.

\section{Author contributions}

JAZ designed the experiments, JAZ, MMB AJA and SM performed the experiments, DWZ analyzed the data, and JAZ and DWZ wrote the ms.

\section{Additional information} Competing financial interests: The authors declare no competing financial interests.

License: This work is licensed under a Creative Commons

Attribution-NonCommercial-ShareAlike 3.0 Unported License. To view a copy of this license, visit http://creativecommons.org/licenses/by-nc-sa/3.0/

How to cite this article: Zeh, J.A., Bonilla, M.M., Adrian, A.J., Mesfin, S. \& Zeh, D.W. From father to son: transgenerational effect of tetracycline on sperm viability. Sci. Rep. 2, 375; DOI:10.1038/srep00375 (2012). 